\title{
Micro-Structured Fiber Interferometer as Sensitive Temperature Sensor
}

\author{
F. C. FAVERO*, M. BECKER, R. SPITTEL, M. ROTHHARDT, \\ J. KOBELKE, and H. BARTELT
}

Institute of Photonic Technology (IPHT), Albert-Einstein-Straße-9, 07745, Jena, Germany

${ }^{*}$ Corresponding author: F. C. FAVERO E-mail: fernando.favero@ipht-jena.de

\begin{abstract}
We report on a fast and sensitive temperature sensor using a micro-structured or photonic crystal fiber interferometer with a high germanium doped fiber core. The wavelength sensitivity for temperature variation was as high as $\Delta \lambda / \Delta T=78 \mathrm{pm} /{ }^{\circ} \mathrm{C}$ up to $500{ }^{\circ} \mathrm{C}$, which was 6 times more sensitive than the fiber Bragg grating temperature sensitivity of $\Delta \lambda / \Delta T=13 \mathrm{pm} /{ }^{\circ} \mathrm{C}$ at $1550 \mathrm{~nm}$. The sensor device was investigated concerning the sensitivity characteristics and response time.
\end{abstract}

Keywords: Micro-structured optical fiber, fiber interferometer, fiber temperature sensor

Citation: F. C. FAVERO, M. BECKER, R. SPITTEL, M. ROTHHARDT, J. KOBELKE, and H. BARTELT, "Micro-Structured Fiber Interferometer as Sensitive Temperature Sensor," Photonic Sensors, DOI: 10.1007/s13320-013-0116-5.

\section{Introduction}

Many different kinds of temperature fiber sensors have been demonstrated by different research groups. A simple structure like a temperature fiber sensor was manufactured using a Fabry-Perot interferometer made between two cleaved sapphire fiber end faces [1]. Nguyen et al. [2] reported a temperature sensor using a combination of multi-single-multimode fibers between single mode fibers with a high temperature sensitivity of $88 \mathrm{pm} /{ }^{\circ} \mathrm{C}$. Villatoro et al. [3] reported a new kind of Mach-Zehnder fiber interferometer where a photonic crystal fiber (PCF) stub was spliced between single mode fibers, and in the splice region, the PCF voids were fully collapsed. Two years later, a Mach-Zehnder fiber interferometer was demonstrated as the temperature sensor, after a long fiber annealing [4], and a temperature sensitivity of about $8 \mathrm{pm} /{ }^{\circ} \mathrm{C}$ was demonstrated. Similar to the interferometer presented here, many other research groups reported on fiber optic sensors to measure temperature. In 2009, Larrion et al. [5] reported an interferometer using a commercial PCF as the temperature sensor, but the low temperature range of operation and the necessity of nanoparticles deposition make the sensor impracticable for outdoor applications. In the same year, Peiró et al. [6] reported a temperature sensor using a suspended core fiber with the germanium doped core, the group filled the fiber with a liquid and found a high temperature sensitivity of $25 \mathrm{~nm} /{ }^{\circ} \mathrm{C}$, and the need to fill the fiber with liquid can make the production slow and increase costs.

Other sensors were reported as temperature sensors, using liquids inside of the PCF voids, such as ethanol [7] or a colloid [8]. The sensitivities found in these cases were $6.6 \mathrm{~nm} /{ }^{\circ} \mathrm{C}$ and $70 \mathrm{pm} /{ }^{\circ} \mathrm{C}$, 
respectively.

However, until now, the devices that use the fiber filled with liquid have only a short operation range. Another temperature sensor reported used a combination of splices between multimode fiber-PCF- and single mode fibers [9], where a temperature sensitivity of $73 \mathrm{pm} /{ }^{\circ} \mathrm{C}$ was demonstrated, but the device needed the 40-cm PCF for its preparation. In this paper, we report on a small and simple fiber interferometer as the temperature sensor which used a PCF with a high concentration of germanium inside its core. In contrast to the papers mentioned above, the device had a short length $(<2.0 \mathrm{~mm})$ and did not require any fiber annealing to operate until $500{ }^{\circ} \mathrm{C}$. The device was designed for operation in reflection. The PCF was spliced between two single mode fibers in order to build an interferometer. In the splicing regions, the PCF voids were fully collapsed while the solid fiber core was conserved. A numerical simulation was performed in order to explain the interference of guided modes in the PCF.

\section{Principle}

A PCF with a specially doped germanium core as shown in Fig. 1 was used to build the interferometer. The fiber core consisted of a double refractive index structure. The highly doped central core with a diameter of $1.2 \mu \mathrm{m}$ was surrounded by a pedestal-like structure with a diameter of $3.4 \mu \mathrm{m}$. As shown in Fig. 1(b), the core had a shaped concentration profile with approximately $32 \mathrm{~mol} \%$ (right side) at its top and $13 \mathrm{~mol} \%$ at the maximum of the pedestal. The PCF specifications are shown in Table 1.

The fiber was spliced between pieces of conventional single mode fibers (SMF). In the splicing region, the PCF voids were fully collapsed, as represented in Fig. 2. In the first splice (SMF $\rightarrow \mathrm{PCF}$ ), the fundamental core mode and higher order cladding modes of the PCF got excited, and the second splice $(\mathrm{PCF} \rightarrow \mathrm{SMF}$ ) had the function of combining the excited modes. The advantage of having a PCF with a high refractive index in the core (germanium doped) is that the light can be guided in the cladding region (in particular in our case due to the device having the fully collapsed region and because the PCF core was smaller than the SMF core). The PCF allows the propagation of different modes in the cladding due to its specific structure as reported in $[10,11]$. In the microstructure cladding region, leaky modes can propagate with a (complex) refractive index below the value of the fundamental space filling mode. This effect is sometimes also called the pseudo-bandgap [12].

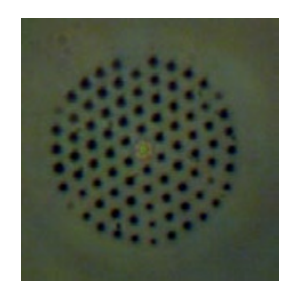

(a)

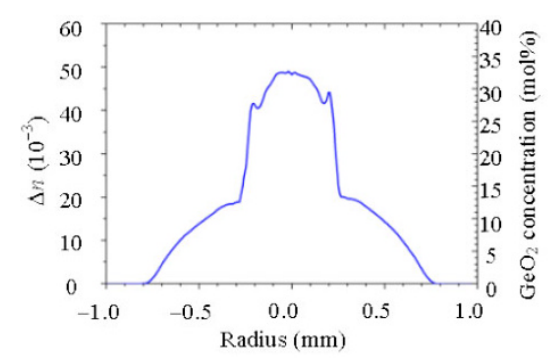

(b)

Fig.1 Refractive index difference as a radius function: (a) the cross section of the microstructure area and (b) measured refractive index and calculated $\mathrm{GeO}_{2}$ concentration profile of the core rod which was used for the PCF fabrication (the left side indicates the variation of the refractive index with the $\mathrm{GeO}_{2}$ concentration in the right side).

Table 1 Comparison between PCF and SMF28 properties.

\begin{tabular}{ccccc}
\hline Fiber & Fi. dia. $(\mu \mathrm{m})$ & Core dia. $(\mu \mathrm{m})$ & Hol. dia. $(\mu \mathrm{m})$ & $\Lambda(\mu \mathrm{m})$ \\
\hline PCF & 124 & $1.2 / 3.4$ & 1.0 & 4.5 \\
SMF & 125 & 8.6 & $/$ & $/$ \\
\hline
\end{tabular}

The power distribution between the interfering modes could be adjusted by an offset of the splice. 
The second splice (PCF $\rightarrow \mathrm{SMF})$ worked as the spatial filter which only collected the light from the PCF core. An interferometer with the same principle of the shifting core position was reported in $[3,10]$.

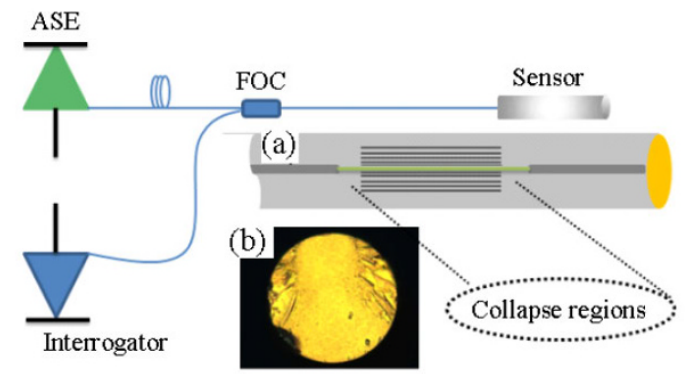

Fig. 2 Experimental setup: (a) head sensor and (b) end fiber with the gold film.

The measured signal was an interference pattern in transmission. However, for practical measurement reasons, a reflection measurement concept is more advantageous. Therefore, we cleaved the second SMF one centimeter after the splice region and deposited a gold film with approximately $50-\mathrm{nm}$ thickness on the fiber end face using a metal evaporation technique.

The gold film operated as a mirror in order to make the device operate in reflection and to improve the reflection signal in comparison to the Fresnel reflection in the silica-air interface (approximately $4 \%)$.

After the gold film deposition, the signal increased about $13 \mathrm{~dB}$ compared to the Fresnel reflection. Considering the operating wavelength around $1550 \mathrm{~nm}$, which was used in the experimental setup, only the fundamental mode could propagate inside the fiber core [see Fig.2(a)]. Nevertheless, the cladding was capable of guiding a high number of modes.

To determine the modes which could interfere with the fundamental core mode, the guided power was used with the first ring of air holes. The overlap of the fundamental mode [see Fig. 3(a)] with the doped core region was $71 \%$, and the guided power within the first ring of air roles was $100 \%$. The defect mode with the highest overlap in the first ring zone [see Fig. 3(b)] carried $6 \%$ of its power in the doped core and $35 \%$ within the first ring of air holes.

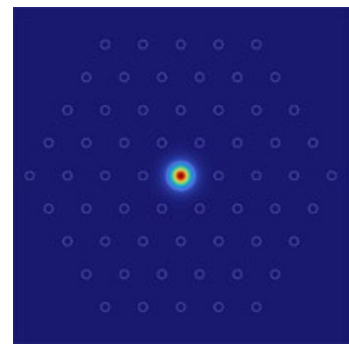

(a)

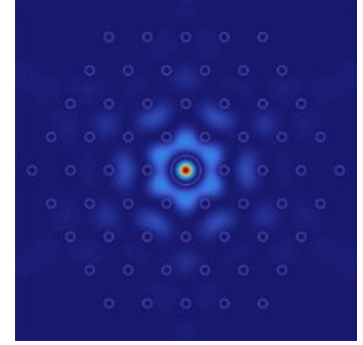

(b)
Fig. 3 Modes that participate in the interference: (a) fundamental core mode and (b) higher-order leaky modes with the strongest overlap in the first ring of roles.

These two modes interfered along their propagation inside the PCF. Generally, the interference of two modes can be described [4] as

$$
I=I_{1}+I_{2}+2 \sqrt{I_{1} I_{2}} \cos (\theta)
$$

where $I_{1}$ and $I_{2}$ are the intensities of the fundamental mode and higher order cladding mode, respectively. $L$ is the length of the PCF stub. The phase difference, $\theta$, can be written as a function of the fiber length, the refractive index difference $\Delta n_{\text {eff }}$ between the two interfering modes $\left(\Delta n_{\text {eff }}=n_{1}-n_{2}\right)$, and the wavelength $\lambda$ :

$$
\theta=\left(\beta_{1}-\beta_{2}\right) \cdot L=\frac{2 \pi}{\lambda} \Delta n_{\mathrm{eff}} L
$$

where $\beta_{1}$ and $\beta_{2}$ are the propagation constants.

The refractive index difference between the modes can be calculated using the space between two consecutive peaks at the wavelengths $\lambda_{1}$ and $\lambda_{2}$ :

$$
\Delta n_{\text {eff }}=\frac{1}{L} \frac{\lambda_{2} \cdot \lambda_{1}}{\lambda_{2}-\lambda_{1}}
$$

The wavelength shifts a minimum of the interference pattern of $\left(\delta \lambda_{n}\right)$ as a function of temperature variation $(\Delta T)$ of a single transmission dip can be written as a function of the refractive index:

$$
\delta \lambda_{1}=2 L \cdot \frac{\lambda_{2}-\lambda_{1}}{\lambda_{2}+\lambda_{1}} \frac{\delta \Delta n_{\text {eff }}}{\delta T} \cdot \Delta T
$$

where $\Delta T$ is the temperature variation, and $\partial \Delta n_{\text {eff }} / \partial T$ is the variation of the effective refractive index difference of the guided modes with the temperature. 
The influence of the thermally induced length change is equal for both modes and does not result in a wavelength shift.

The value of the refractive index variation with the temperature depended strongly on the germanium doping level and was considerably higher than that for un-doped silica $[6,7]$.

The thermo-optical coefficient of pure silica was $1.06 \times 10^{-5} \mathrm{~K}^{-1}[13]$. The effect of germanium doping on the thermo-optical coefficient was reported in [14]. From these results, we could approximate a molar $\mathrm{GeO}_{2}$ concentration slope on the thermo-refractive index of about $1.2 \times 10^{-7} \mathrm{~K}^{-1} \mathrm{~mol} \%{ }^{-1}$. A variation of $15 \%$ Ge doping between the central core and the socket would result in a difference of the temperature coefficient of the modes of about $\partial n_{\text {eff }} \partial T=1.6 \times 10^{-6} \mathrm{~K}^{-1}$. The device had a length of $1.80 \mathrm{~mm}$ and a period of $\lambda_{2}-\lambda_{1}, 44.39 \mathrm{~nm}$. From these data, a wavelength sensitivity of $83 \mathrm{pm} / \mathrm{K}$ can be expected theoretically.

A temperature difference of $500 \mathrm{~K}$ would therefore result in an interference pattern shift of about $41 \mathrm{~nm}$.

\section{Sensor packaging and measurement}

The fiber was packaged inside the metal tubing with the outside diameter of $1.0 \mathrm{~mm}$ and the inside diameter of $400 \mu \mathrm{m}$ (see Fig. 4) with the alumina ceramic glue 989 , which resisted up to $1500^{\circ} \mathrm{C}$.

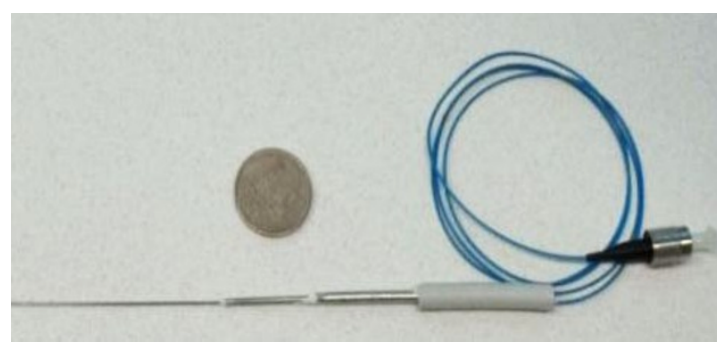

Fig. 4 Picture of the encapsulated sensor with the metal tube and $\mathrm{PC}$ connector next to a $50 \mathrm{c}$ coin.

Such an arrangement as shown in Fig. 4 assures the good mechanical stability. The device was tested in an oven for a temperature range between the room temperature and $500{ }^{\circ} \mathrm{C}$, with the heating and cooling rate of $10{ }^{\circ} \mathrm{C} / \mathrm{min}$.

The temperature was monitored with a thermocouple PT100 placed next to the fiber sensor.

For illumination of the sensor, the broadband source (ASE) was used, and the details of the temperature were acquired with an optical spectrum analyzer.

The result is shown in Fig. 5. The inset of Fig. 5 is the reflection spectrum of the interferometer before (black dots) and after (upper line) the gold deposition in the end of the fiber.

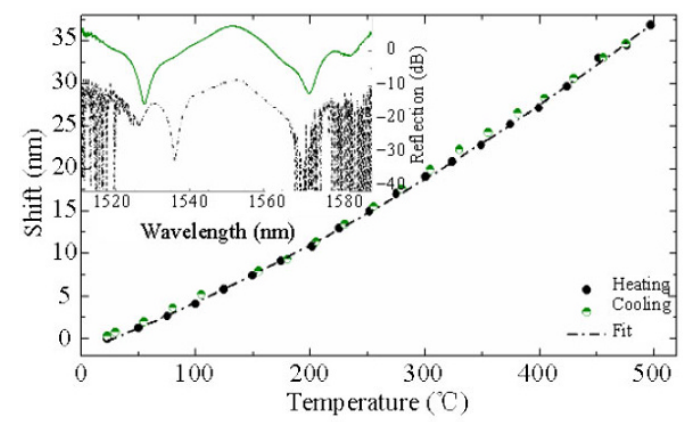

Fig. 5 Results of the temperature test up to $500^{\circ}$ (the sensitivity measured is $78 \mathrm{pm} /{ }^{\circ} \mathrm{C}$ ).

The total wavelength shift with the temperature $(\Delta \lambda / \Delta T)$ was measured as $78 \mathrm{pm} /{ }^{\circ} \mathrm{C}$. This value was about 6 times higher than that for a fiber Bragg grating. A fiber Bragg grating in the same fiber achieved a thermal sensitivity of $13 \mathrm{pm} /{ }^{\circ} \mathrm{C}$, as shown in Fig. 6.

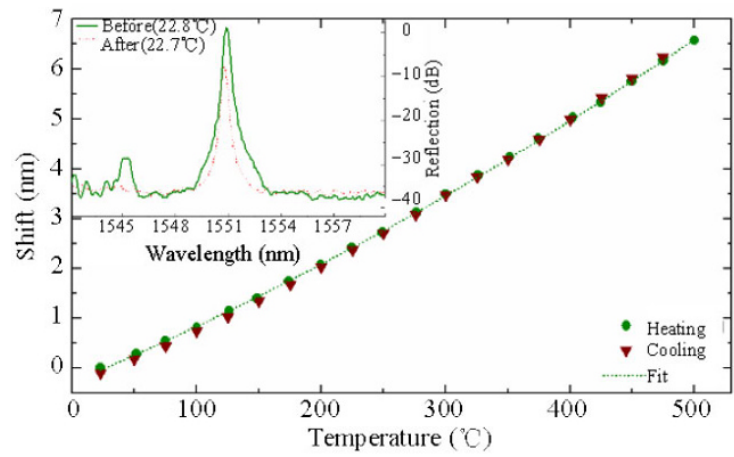

Fig. 6 Results of the temperature test up to $500{ }^{\circ} \mathrm{C}$ in an FBG written in the PCF [the dots represent heating, the triangles represent cooling, and the figure inset is the reflection spectrum of the FBG before (dots) and after (upper line) the gold deposition in the thermal cycle]. 
As for an additional parameter, the response time for a temperature difference between the room temperature $\left(\sim 22^{\circ} \mathrm{C}\right)$ and high temperature of $500{ }^{\circ} \mathrm{C}$ in the oven was investigated.

The device was put in the oven and removed after 25 seconds approximately. The spectral sensor responses of both sensor types were measured. Figure 7 shows the wavelength shifts of the PCF interferometer (upper line) and the FBG (lower line), which were measured with a commercial FBG interrogator (IBSEN I-MON E-USB 2.0). The temperature response time of about $3.0 \mathrm{~s}$ from the room temperature to the high temperature for both cases (PCF interferometer device and FBG device) was measured, and the temperature response time of the high temperature to low temperature of about $14.0 \mathrm{~s}$ was measured. These are comparable values for both types of the sensor and attractive parameters for commercial applications.

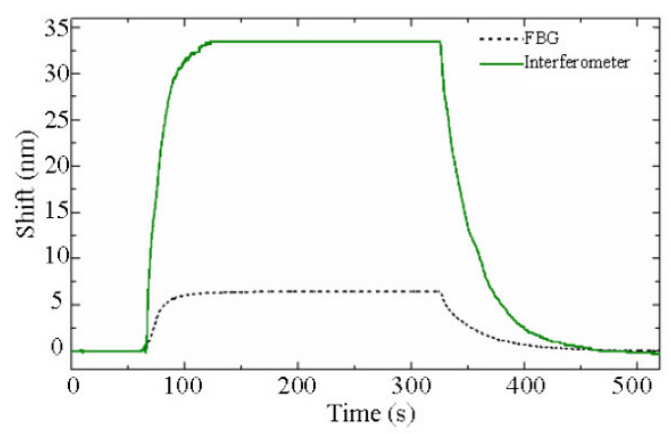

Fig. 7 Measurements of the response time, from the room temperature (about $22{ }^{\circ} \mathrm{C}$ ) to the maximum temperature up to $500{ }^{\circ} \mathrm{C}$.

\section{Conclusions}

We demonstrated the use of a new type of structured optical fiber in a modal interferometer for a sensitive temperature measurement. A gold film deposition was made at the end of the SMF in order to operate the device in reflection. The temperature dependence had a high temperature sensitivity of $78 \mathrm{pm} /{ }^{\circ} \mathrm{C}$. A package suitable for the high temperature measurement has been presented. The small size, the possibility of using reflected signals and the high temperature stability up to more than $500{ }^{\circ} \mathrm{C}$ make such a sensing element applicable for harsh environment conditions.

\section{Acknowledgment}

Funding by the Thuringian Ministry of Education, Science and Culture and the European EFRE program is gratefully acknowledged.

Open Access This article is distributed under the terms of the Creative Commons Attribution License which permits any use, distribution, and reproduction in any medium, provided the original author(s) and source are credited

\section{References}

[1]M. Willsch, T. Bosselmann, P. Flohr, R. Kull, W. Ecke, I. Latka, et al., "Design of fiber optical high temperature sensors for gas turbine monitoring," in Proc. SPIE, vol. 7503, pp. 75037R-1-75037R-4, 2009.

[2] L. V. Nguyen, D. Hwang, S. Moon, D. S. Moon, and Y. Chung, "High temperature fiber sensor with high sensitivity based on core diameter mismatch," Optics Express, vol. 16, no. 15, pp. 11369-11375, 2008.

[3] J. Villatoro, V. Finazzi, V. P. Minkovich, V. Pruneri, and G. Badenes, "Temperature-insensitive photonic crystal fiber interferometer for absolute strain sensing," Applied Physics Letters, vol. 91, no. 9, pp. 091109-1-091109-3, 2007.

[4] G. Coviello, V. Finazzi, J. Villatoro, and V. Pruneri, "Thermally stabilized PCF-based sensor for temperature measurements up to $1000{ }^{\circ} \mathrm{C}$," Optics Express, vol. 17, no. 24, pp. 21551-21559, 2009.

[5] B. Larrión, M. Hernánez, F. J. Arregui, J. Goicoechea, J. Bravo, and I. R. Matías, "Photonic crystal fiber temperature sensor based on quantum dot nanocoatings," Journal of Sensors, vol. 2009, 932471-1-932471-6, 2009.

[6] S. Torres-Peiro, A. Diez, J. L. Cruz, and M. V. Andres, "Temperature sensor based on Ge-doped microstructured fibers," Journal of Sensors, vol. 2009, pp. 417540-1-417540-5, 2009.

[7] W. Qian, C. Zhao, C. Chan, L. Hu, T, Li, W. Wong, et al., "Temperature sensing based on ethanol-filled photonic crystal fiber modal interferometer," Sensors 
Journal, vol. 12, no. 8, pp. 2593-2597, 2012.

[8] A. Bozolan, R. M. Gerosa, C. J. S. de Matos, and M. A. Romero, "Temperature sensing using colloidal-core photonic crystal fiber," Sensors Journal, vol. 12, no.1, pp. 195-200, 2012.

[9] S. M. Nalawade and H. V. Thakur, "Photonic crystal fiber strain-independent temperature sensing based on modal interferometer," IEEE Photonics Technology Letters, vol. 23, no. 21, pp. 1600-1602, 2011.

[10] Q. Wu, Y. Semenova, J. Mathew, P. Wang, and G. Farrell, "Humidity sensor based on a single-mode hetero-core fiber structure," Optics Letters, vol. 36, no. 10, pp. 1752-1754, 2011.

[11] H. Y. Choi, M. J. Kim, and B. H. Lee, "All-fiber
Mach-Zehnder type interferometers formed in photonic crystal fiber," Optics Express, vol. 15, no. 9, pp. 5711-5719, 2007.

[12] K. Busch and S. John, "Photonic band gap formation in certain self-organizing systems," Physical Review E, vol. 58, no. 3, pp. 3896-3907, 1998.

[13] G. Ghosh, M. Endo, and T. Iwasaki, "Temperature-dependent Sellmeier coefficients and chromatic dispersions for some optical fiber glasses," Journal of Lightwave Technology, vol. 12, no. 8, pp. 1338-1342, 1994.

[14] Y. J. Kim, U. C. Paek, and B. H. Lee, "Measurement of refractive-index variation with temperature by use of long-period fiber gratings," Optics Letters, vol. 27, no. 15 , pp. $1297-1299,2002$. 